\title{
Zeitgeist: Window to your mind
}

\author{
Oliver Mag Gingrich \\ National Centre for Computer \\ Animation, London, UK \\ olivergingrich@gmail.com
}

\author{
Shama Rahman \\ NeuroCreate / Artist \\ London, UK \\ Rahman.shama@gmail.com
}

\begin{abstract}
Zeitgeist consists of an innovative neuro-art piece, which offers to induce Flow states through immersive experiences: Zeitgeist, a new artwork by Dr. Shama Rahman and Dr. Oliver Gingrich is an Al-based digital art piece that explores audiovisual neurofeedback strategies to train 'Flow' to improve cognitive performance and engagement. This exploration will lead to the development of a prototype platform for cognitive enhancement. This art project looks at effective strategies to enhance mental states by inviting the audience to induce Flow through introspection, thereby designing their own internal to external spaces. The team will develop a real-time 'Flow' mental state digital platform utilising EEG deep-learning classification for a feedback loop involving embodied interactive collaborative participation by the audience.
\end{abstract}

Brainwave art. Neuro art. Participatory art. Human computer interface.

\section{INTRODUCTION}

Creative-thinking has shown to increase brain blood flow with direct links to neuronal wellbeing (Chapman 2016). Al, specifically deep-learning for this project, provides a hitherto more accurate classification mode for linking complex brainwave patterns in real-time to a desired visual output, thereby influencing digital interface interaction through neurofeedback. This activity investigates the use of visual neurofeedback, employing creative cognitive frameworks and collaborative creative engagement.

Our project examines visual strategies for neurofeedback entrainment to actively induce 'Flow' mental states. This project is a collaboration with Dr. Shama Rahman, neuroscientist and founder of NeuroCreate, and Dr. Oliver Gingrich from National Centre for Computer Animation (NCCA) at Bournemouth University, concentrating on artistic visualisation, human-centred design and gamification. The paper describes the development of the prototype, artistic influences and discusses future design strategies, applications and the vision for the Zeitgeist 'prototype'.

For the last two years, the research team has worked on the conceptual development for a multisensory application that combines innovative research within cognitive training, to integrate entrainment of Flow, with state-of-the-art artificial intelligence, real-time visualisation and know-how on perception and presence research. NeuroCreate under their founder and CEO, the researcher and artist Dr. Shama Rahman specialises in creative training using novel neurofeedback techniques, utilising deep-learning. Dr. Oliver Gingrich's research at the National Centre for Computer Animation focuses primarily on immersive technologies, visual sound art and co-factors of presence. Together, the team is now working on a prototype to combine elements of their research into an artistic installation that results in collaborative participation using sound and light for the audience, with the potential of cognitive enhancement.

\section{FLOW AND CREATIVE ENGAGEMENT}

Our project implements strategies to actively induce 'Flow' peak performance mental states, which improves mental flexibility and resilience, increase motivation (Veenhoven 1984), meaningful engagement and absorption (Csikszentmihalyi, 1990), and improved mood and happiness (Diener 2000; Diener \& Diener 1996). This leads to peak cognitive performance towards improved wellbeing and reduced stress.

This platform will incorporate visual neurofeedback (combination of EEG classification and visual entrainment), within creative cognitive frameworks and creative thinking tools utilising collaborative creative engagement, multi-sensory integration, as well as simultaneous narrow and broad attention. Innovation-training (also known as creative- 
thinking) has shown to increase brain blood flow by $8 \%$ with direct links to improved neuronal wellbeing (Chapman 2016).

The team will explore the strategy of neurofeedback to train 'Flow'. This form of cognitive training can improve mental performance and wellbeing by non-invasively harnessing our neuroplastic learning. Applying know-how at Bournemouth's NCCA in real-time environments (Gingrich 2016) we will enhance visual entrainment through gamified positive 'nudging' via visual cues (colour, depth, multi-sensory integration, and spatial attention pattern variances).

To build on the efficacy, our novel proposal is a close-knit integration of NeuroCreate's existing neurofeedback products and new collaborative audio-visual strategies to create a real-time Flowtraining participatory art installation, which improves neuro-cognitive performance. Attaining Flow states is a proactive approach to improve resilience via neuroplasticity to actively mitigate the processes underpinning stress and its effects. Relaxation is a more passive approach that deals with the symptoms of stress rather than modifying its underlying neuronal mechanisms.

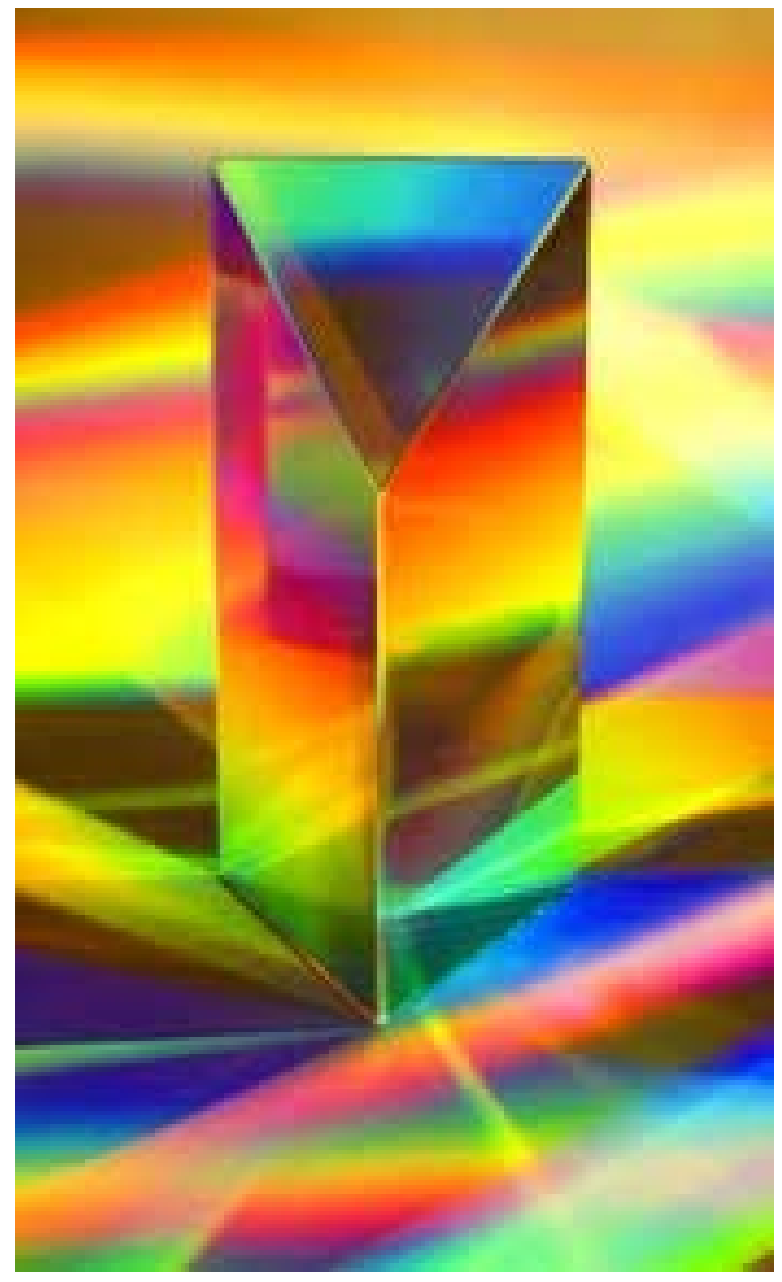

Figure 1: Prism and light refractions.

\section{FLOW, AGILE THINKING AND CREATIVE PRODUCTIVITY}

In her PhD research (Rahman 2014), NeuroCreate's Dr. Rahman identified a signature EEG brainwave pattern for creative Flow. Her findings were supported by $\mathrm{FMRI}$ research on musicians/rappers (Limb \& Braun 2008; Braun et al. 2012). This is exciting evidence that neuronal networks underlying Flow, are common across different domains, irrespective of whether people are engaged in speaking words with mental conceptualisation, or in specialist motor skills. We propose to classify real-time EEG patterns with deep-learning algorithms to indicate when users' Flow states are reached. NeuroCreate's offline algorithm (FlowCreate ${ }^{\mathrm{TM}}$ Profiler) has significant accuracy scores on her $\mathrm{PhD}$ data of 0.8 to 0.9 (0.5 probability is due to chance).

Research shows creative cognition engagement reduces cognitive decline and optimises cognition throughout adulthood (Chapman 2016; McHugh 2016; Bell 2010, 2010; Park et al. 2007; Fancourt and Steptoe 2018). Innovation-thinking and creative cognition frameworks are a mechanism underpinning achieving Flow states (Rahman 2014). It is novel to have digital facilitation of creative cognition for Flow enablement, improved cognitive performance and mental wellbeing.

This is an improvement on other neurofeedback studies on chronic stress (Blaskovitz et al.). Flow entails spatially and temporally more complex brainwave patterns to be classified than relaxation/focus mental states, with novel techniques of deep-learning that are capable of more accurately monitoring these complex patterns. Through this participatory art piece, we propose to operate within a controlled environment, designed within a creative cognition framework.

Utilising this platform, we aim to increase creative engagement and collaboration for audiences to be immersed in a naturalistic creative generation setting (Moneta 2016: "The problem with flow is the difficulty to find appropriate (experimental) conditions"). Improved creative productivity and mental performance leads to higher agility, and less mental fatigue. In addition to cognitive agility, Flow states further mitigate stress by improving engagement and joy. A wellbeing culture survey states there is $40 \%$ reduction in health-related issues when people feel happy (in their work) compared to feeling stressed" (Chapman 2015). 


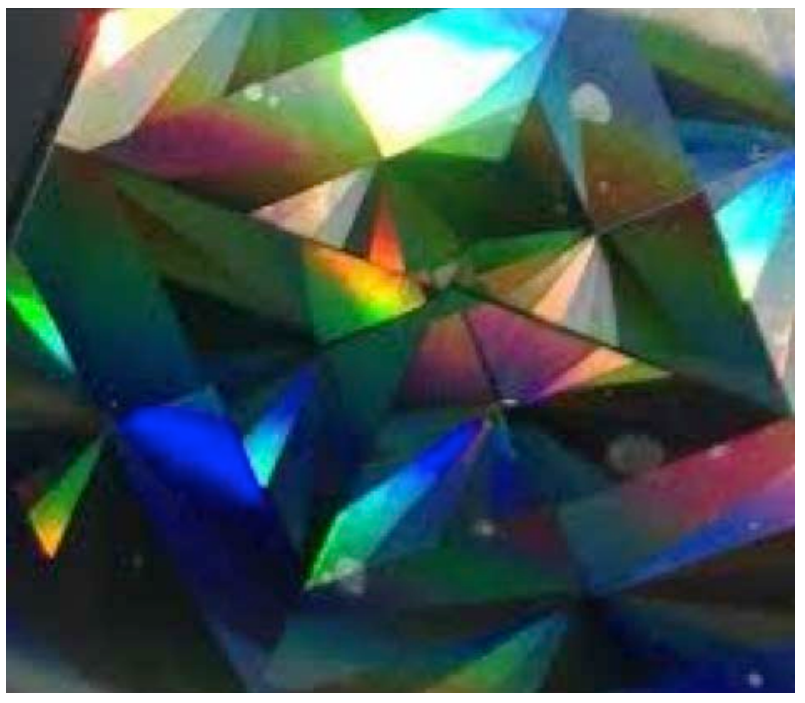

Figure 2: Light refractions.

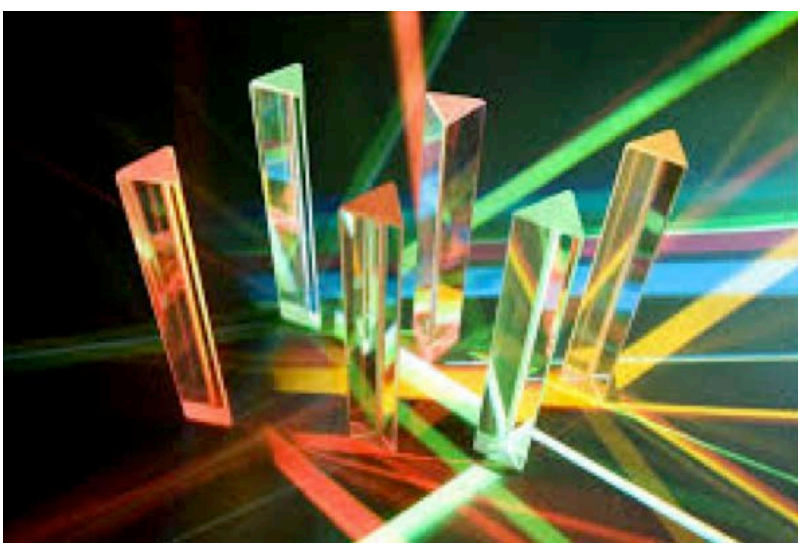

Figure 3: Prismatic Light Refractions.

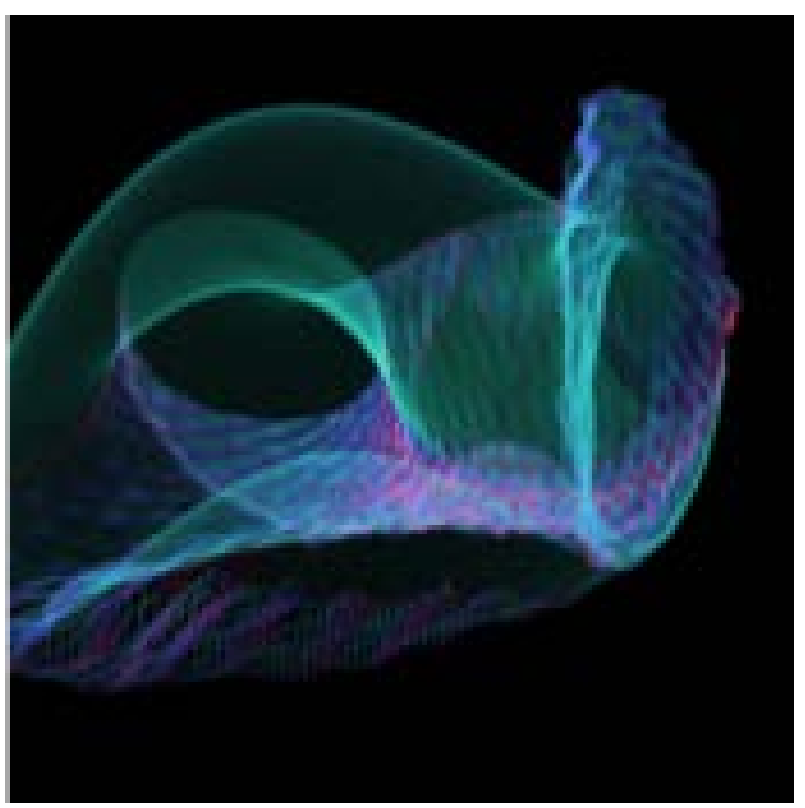

Figure 4: Visual reference Fabio Galicia, 2016 Bartlett.

\section{PROTOTYPE CONCEPT DESCRIPTION}

The installation consists of a central cylindrical clear prismatic totem that displays an interactive digital visualisation, responding to participants' brain activity. Light sources reflect and refract a light beam emitted from above the totem, reacting in real-time to participants' brain activity interaction. The totem itself is filled with glass prisms, from recycled glass bottles.

This light beam concentrates inside the totem as a focal point and subsequently radiates light patterns outwards like a sundial (which forms the basis of our concept 'Zeitgeist'). A secondary component is the light ring above the totem that is contributing to the ambient lighting environment. The ring is emitting light towards the surrounding walls of the space and immersing the participants in a bath of light, thereby aiding their peripheral perception.

The simultaneous multi-modal integration of focal and peripheral attentional cues is a characteristic of Flow states. The visual interaction between focussed and peripheral light sources is determined by participants' own brain activity and importantly the ability to reach Flow states not only independently individually but collaboratively together. These Flow states will be reached through playful creative activities with each other, such as music-making with percussion and humming, movement, poetry slams, painting and drawing. The totem becomes their creative interface.

\section{TECHNICAL DESCRIPTION}

The Zeitgeist installation is powered by three computers - one specifically running a TouchDesigner script that receives as input, 'Flow state classifications' from Neurocreate's proprietary deep-learning pipeline. Each of the three computers will host a local copy of the deeplearning algorithm which will be fed by Bluetooth enabled LSL protocols from 3 corresponding Muse headsets. TheseMuse headsets provide real-time streaming EEG brain activity data of participants. This new streaming data set is compared to a training dataset collated and verified by NeuroCreate, analysing how likely a participant is in Flow at any given time on a continuous scale of 0 to 1.

The TouchDesigner patch receives this sliding scale input, and translates this Flow participant data in real-time into DMX messages. DMX messages are a light protocol used to trigger LED lights. We are mapping both colours and brightness to the intensity of the Flow state parameters provided by our participants in real-time. Individual 


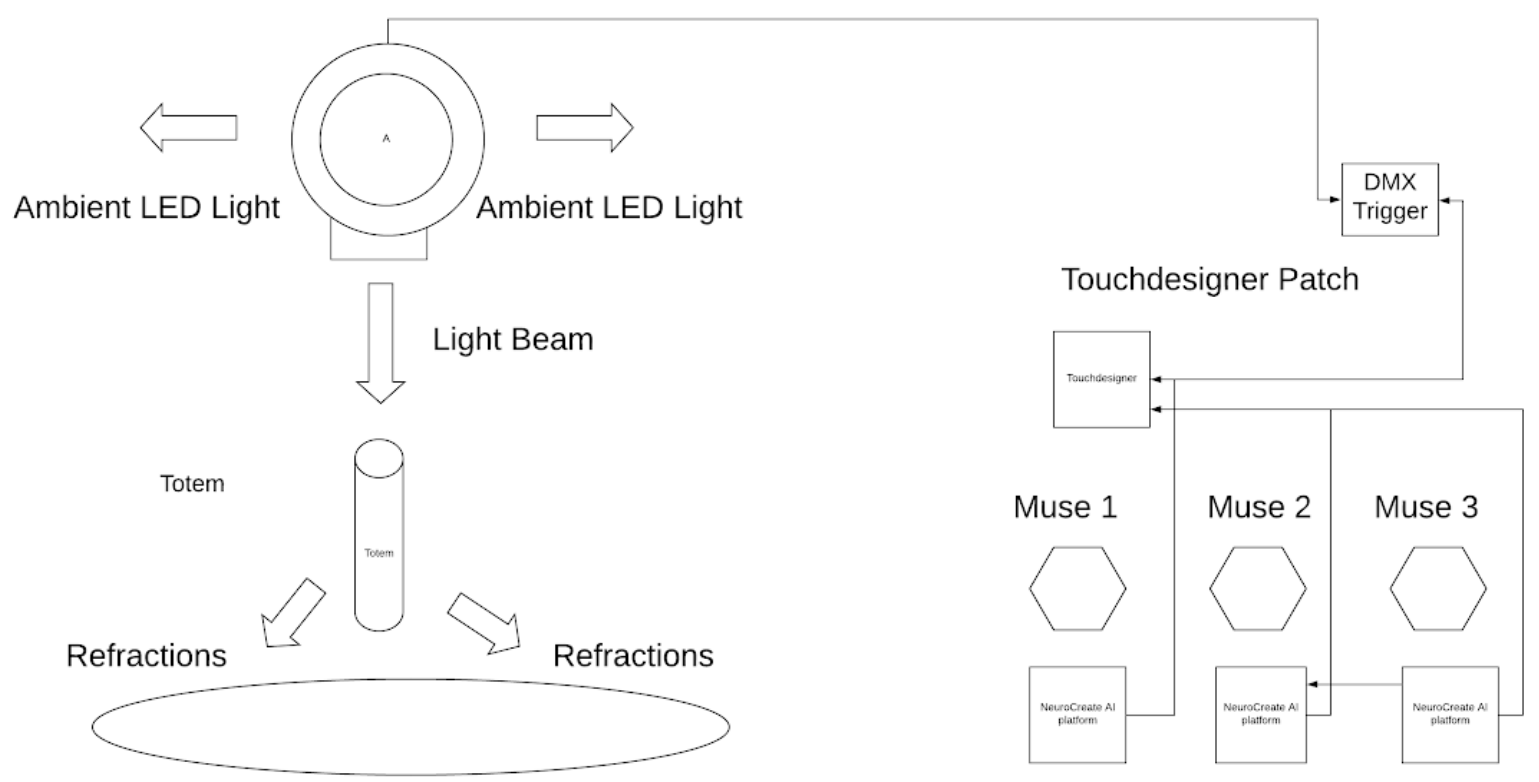

Figure 5: Technical Diagram - Zeitgeist.

participants' Flow states will influence the central focal point lights i.e. the colour hue, saturation and brightness. Collaborative achievement of Flow states will influence ambient peripheral lighting. This gamification of Flow, the collective goal to achieve Flow states together, turns the piece into a collaborative creative canvas, a shared space, in which every individual can contribute through their own creative potential.

\section{CRITICAL ANALYSIS}

This visual neurofeedback loop will trigger Flow states, where audiences will be encouraged to engage in creative play with one another. Initially, we are planning to take the installation to several media art exhibitions, including a presentation at EVA London and ART IN FLUX's exhibition at the Computer Arts Society's headquarters in Moorgate. The artwork will be designed to be at once modular and site specific, so we can adapt it depending on the location of the exhibition. We would be interested to explore the potential of these narrow and broad simultaneous cues to entrain Flow states, through further research, ideally combining questionnaires and surveys, with an analysis of the efficacy of such an interface for creative Flow enablement.
Furthermore, we are actively planning the next stage of this prototype, namely to create a cylindrical holographic display with different brainwave visualisations for different participants such as geometric abstract patterns and shapes (Figure 5 and Figure 7). The future of this prototype development, might also see more therapeutic, academic or digital commercial applications such as through integration with Neurocreate's other existing product, the FlowCreate ${ }^{\mathrm{TM}}$ Innovator.

NeuroCreate's Al creative collaborator sparks human creativity by inspiring, expanding and analysing ideas within strategy and content creation. It is a visualised 'thinking' tool that trains creative-thinking and augments the creative process. By digitising design-thinking tools and providing a flexible digital innovation framework, this tool guides learners through different stages of creative cognition. Its initial principle focuses on creative stimulation through the concept of active semantic seeding using words as symbols of thought.

The Innovator uses the principals of 'neurodesign' (design informed by the underlying neurocognitive mechanisms) to lead to 'augmented intelligence' for users. This third development phase could see the to integration of neurofeedback with gamified visual strategies honed and explored through this art installation. It would result in yet another more digitally scaleable mode of this art piece. 


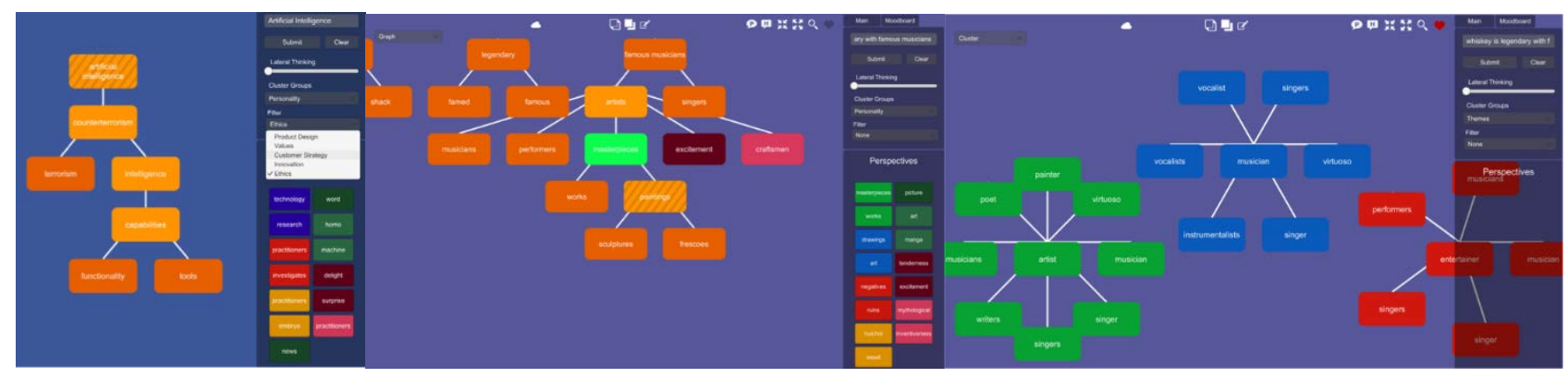

Figure 6: NeuroCreate's Innovator.

\section{CONCLUSION}

Zeitgeist presents a novel interface for collaborative creative engagement, a tool to actively induce flow states, states of heightened creative activity. As a participatory environment, Zeitgeist is conceptualised to be versatile and modular, and adaptable for a variety of spaces and contexts. Iterations of the project focusing on collaborative music creation are as conceivable as poetry slam environments, or collaborative dance improvisations.

The piece is egalitarian, in that all participants contribute to the experience at equal measures, and it turns the audience into a performer in their own right. The combination between $\mathrm{Al}$, participatory art, and brainwave human computer interface is in itself unique, fusing the latest development in brainwave research with the potential of machine learning.

The artwork has the potential to double up as an art work, while collecting data on audience interaction, helping to understand the full creative potential of participatory arts better. This focus on collaborative art forms, in the service of wellbeing, social connectedness and creative engagement forms part of a wider discourse on the societal impact of media arts.

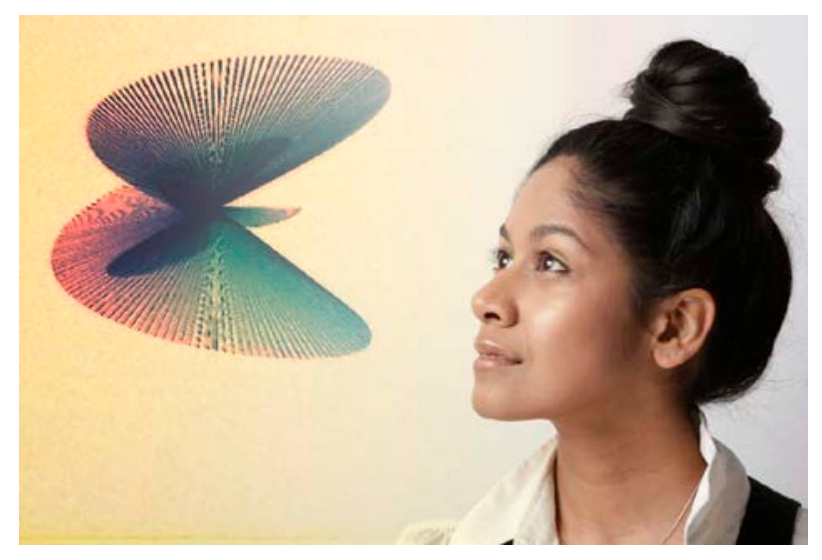

Figure 7: Zeitgeist and Previsualisation.

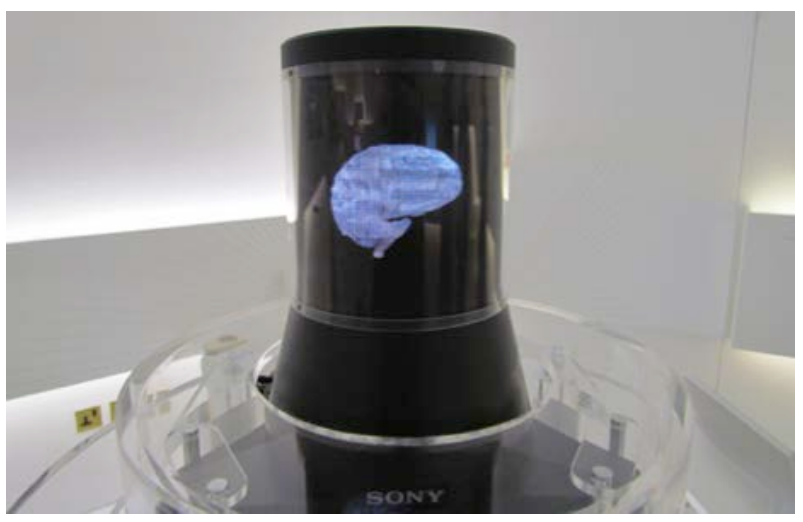

Figure 8: Cylindrical Hologram.

\section{REFERENCES}

Bell, C. C. (2010) Promoting Creative Engagement in the Elderly. Clinical Psychiatry News.

Braun, A. R., Liu, S., Chow, H. M., Erkkinen, M. G., Swett, K. E., Eagle, M.W. and Rizik-Baer, D. A. (2012) Neural Correlates of Lyrical Improvisation: An fMRI Study of Freestyle Rap. Scientific Reports, Nature.

Chapman, B. and Sisodia, R. (2015) Everybody Matters: The Extraordinary Power of Caring for your people like Family. New York: Portfolio.

Chapman, S. B., Aslan, S., Spence, J. S., Keebler, M. W., DeFina, L. F., Didehbani, N., Perez, A. M., Lu, H., and D'Esposito, M. (2016) Distinct Brain and Behavioral Benefits from Cognitive vs. Physical Training: A Randomized Trial in Aging Adults. Frontiers in Human Neuroscience.

Csikszentmihalyi, M. (1990) Flow: The Psychology of Optimal Experience. Journal of Leisure Research, 24(1), pp.93-94

Diener, E. and Diener, C. (1996) Most people are happy. Psychological Science, 7(3), pp.181-185.

Diener, E. (2000) Subjective Wellbeing The science of happiness and a proposal for a national index. American Psychologist, 55(1), pp.34-43. 
Fancourt, D., and Steptoe, A. (2018) Cultural engagement predicts changes in cognitive function in older adults over a 10 year period: findings from the English Longitudinal Study of Ageing. Scientific Reports 8, Nature.

Gingrich, O. (2016) Evoking presence through creative practice on Pepper's ghost displays. PhD Thesis. Bournemouth University, Faculty of Media and Communication.

Gingrich, O., Renaud A., Emets, E., and Aura, A. (2019) Aura: A holographic brainwave interface. EVA London 2019 (Electronic Visualisation and the Arts). doi: 10.14236/ewic/EVA2018.66

Limb, C. J., and Braun, A. R. (2008) Neural Substrates of Spontaneous Musical Performance: An fMRI Study of Jazz Improvisation. PLOS ONE 3(2): e1679.
McHugh, M. C. (2016) Experiencing Flow: Creativity and Meaningful Task Engagement for Senior Women, Women and Therapy, 39.

Moneta, G. B. (2016) Validation of the short flow in work scale (SFWS). Personality and Individual Differences 109 (2017) pp.83-88. doi: 10.1016/j.paid.2016.12.033

Park et al. (2007) An Engagement Model of Cognitive Optimization Through Adulthood. The Journals of Gerontology: Series B, 62, Special Issue 1, June 2007.

Rahman, S. (2014) The neuroscience of musical creativity using complexity tools. PhD Thesis, Imperial College London.

Veenhoven, R. (1984) Conditions of Happiness. Springer, Netherlands. 